\title{
Structural stability of topologically close-packed phases: Understanding experimental trends in terms of the electronic structure
}

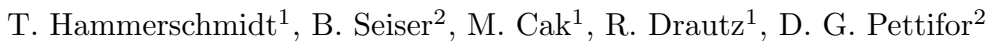 \\ ${ }^{1}$ Atomistic Modelling and Simulation, ICAMS, Ruhr-Universität Bochum, D-44801 Bochum, Germany \\ ${ }^{2}$ Department of Materials, University of Oxford, Parks Road, Oxford OX1 3PH, United Kingdom
}

Keywords: topologically close-packed phases, density-functional theory, tight-binding, bond-order potential, structure map

\begin{abstract}
Topologically close-packed (TCP) phases in single crystal Ni-based superalloys have a detrimental effect on the mechanical properties. In order to gain a microscopic understanding of the factors that control TCP phase stability, we carry out atomistic calculations based on the electronic structure. In particular, we use a hierarchy of methods that treat the electronic structure at different levels of coarse-graining, i.e. at different levels of computational cost and accuracy.

The applied levels of approximation range from density functional theory (DFT) to tight-binding (TB) to bond-order potentials (BOPs). This hierarchy of electronic structure methods allows us to interpret the findings of a recently derived structure map of experimentally observed TCP stability. The TB and BOP calculations are compared to extensive high-throughput DFT calculations for the TCP phases A15, C14, C15, C36, $\mu, \sigma$, and $\chi$ of transition-metal elements.

These findings are extended to binary systems based on DFT heat-of-formations for TCP phases in the systems $\mathrm{V} / \mathrm{Nb}-\mathrm{Ta}, \mathrm{Nb} / \mathrm{Mo}-\mathrm{Ru}, \mathrm{V} / \mathrm{Cr} / \mathrm{Nb} / \mathrm{Mo}-\mathrm{Re}, \mathrm{V} / \mathrm{Cr} / \mathrm{Nb} / \mathrm{Mo}-$ Co. By pairwise comparisons of selected systems, we illustrate the interplay of the difference in average valenceelectron concentration $\bar{N}$ and the composition-dependent relative volume difference $\overline{\Delta V / V}$. Such an approach could be useful to predict the change of expected TCP phase stability due to changes of the composition for a given multi-component alloy.
\end{abstract}

\section{Introduction}

Topologically close-packed (TCP) phases [1] play an important role in modern alloys and steels. While particular TCP phases are desirable in precipitate-hardened steels, the precipitation of TCP phases in single crystal Ni-based superalloys has a detrimental effect on the mechanical properties. The formation of these phases in Ni-based superalloys [2] is attributed to high local concentrations of refractory elements. These elements are used in Ni-based superalloys to increase creep resistance (Mo, Re, W) and to retard the coarsening of the $\gamma^{\prime}$ phase (Re). A more detailed understanding of the thermodynamic stability of TCP phases and their formation will therefore be beneficial for the design of the next generation of superalloys.

The experimentally observed stability of TCP phases in transition metal compounds is to a large extent driven by the average valence-electron concentration and a composition-dependent size-factor difference [3]. A recent analysis of available experimental data in terms of a two-dimensional structure map (Fig. 1) highlighted the well-known fact that TCP phases are typically observed for half-filled d-band (see e.g. Ref. [1]). The coordinates used in the structure map are the average valence-electron concentration

$$
\bar{N}=\sum_{i} c_{i} N_{i}
$$

and a composition-dependent relative volume difference

$$
\overline{\Delta V / V}=\sum_{i, j} c_{i} c_{j}\left|V_{i}-V_{j}\right| /\left[\left(V_{i}+V_{j}\right) / 2\right]
$$

with the concentration $c_{i}$, the number of valence electrons $N_{i}$ and the atomic volume $V_{i}$ of constituent $i$. The structure map also shows that the regions of TCP stability deduced from binary systems (polygonal areas in Fig. 1) are transferable to multi-component systems [3] described by the corresponding $\bar{N}$ and $\overline{\Delta V / V}$. This in turn is the justification for reducing the investigation of TCP phases in the combinatorially complex Ni-based superalloys to the study of binary transition-metal systems.

In this work, we combine a theory-guided analysis of the available experimental data represented in the structure map [3] with the results of a hierarchy of theoretical electronic-structure methods in order to gain a detailed understanding of the microscopic factors that control the stability of TCP phases [4]. Our simulation methods range from high-throughput density-functional theory calculations to canonical tight-binding models and and 


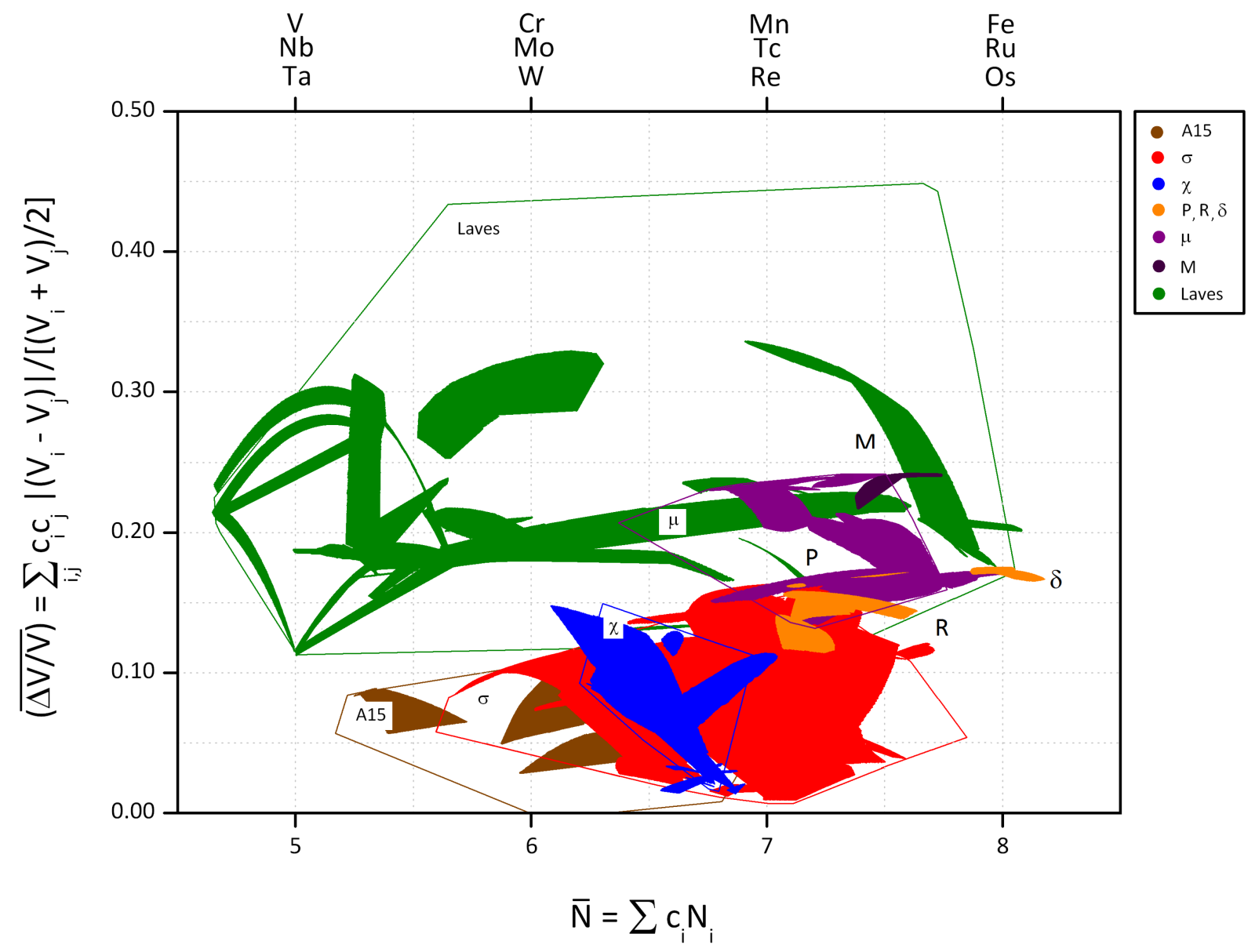

Figure 1: Factors that influence TCP phase stability in binary transition-metal alloys: The average valence-electron concentration $\bar{N}$ and composition-dependent size-factor difference $\overline{\Delta V / V}$ of the TCP structure map. (Reprinted from Acta Materialia 59, B. Seiser, R. Drautz and D.G. Pettifor, TCP phase predictions in Ni-based superalloys: Structure maps revisited, p. 749-763, Copyright (2011), with permission from Elsevier.)

molecular-dynamic simulations with analytic bond-order potentials.

The following sections are organised as follows: After outlining the methodology in the second section, we collect the predictions of the empirical structure map for a set of exemplary binary compounds with different average valence-electron concentration $\bar{N}$ and compositiondependent relative volume difference $\overline{\Delta V / V}$ in the third section. The fourth section is devoted to a discussion of effects driven by the average valence-electron concentration $\bar{N}$ in terms of tight-binding models and bond-order potentials. In the last section we discuss effects arising from the composition-dependent relative volume difference $\overline{\Delta V / V}$ by comparing density-functional theory calculations for TCP phases in several binary transition-metal (TM) systems.

\section{Methodology}

In the following sections we apply a hierarchy of electronic structure methods in order to understand the trends in the experimentally observed structural stability of the TCP phases.

The DFT results presented below are obtained by 
self-consistent total-energy calculations with the VASP code $[5,6,7]$ using projector augmented wave (PAW) pseudopotentials [8] and the local-density approximation (LDA) to the exchange-correlation functional. Our DFT calculations are converged to below $1 \mathrm{meV} /$ atom in the energy differences by using a plane-wave cutoff of $400 \mathrm{eV}$ and a density of the Monkhorst-Pack k-point mesh [9] of $0.020 \AA^{3}$. In all DFT calculations we fully relaxed the simulation cells including internal degrees of freedom and determined the equilibrium lattice constant(s) by a numerical fit to the Birch-Murnaghan equation-ofstate $[10,11]$. An inevitable uncertainty in our results is the choice of the exchange-correlation functional. In similar DFT calculations for TCP phases in the systems Re-V and Re-Ta, we found that the variation of the heat-offormation due to the choice of exchange-correlation functional is of similar order of magnitude as the contribution of the configurational entropy to the free energy at elevated temperatures. The resulting qualitatively different TCP phase stability at elevated temperatures will be discussed elsewhere [12].

For the analysis of the effect of the average valenceelectron concentration $\bar{N}$ on the structural stability of TCP phases we furthermore employ simplified models of the electronic structure. We use an analytic bond-order potential (BOP) that is derived by systematically coarsegraining the electronic structure from density-functional theory to tight-binding (TB) to bond-order potentials (BOP), see e.g. Ref. [13] for a tutorial introduction. The TB model and the analytic BOP [14] for transition metals depend explicitly on the number of valence electrons. In the following we will use the BOP formalism with a canonical TB model $[15,16]$ for understanding qualitative trends in the structural stability. Recently developed material-specific TB models [17] are employed in first molecular-dynamic simulations of interfaces between cubic phases and TCP phases. The tight-binding and bond-order potential calculations presented in this work were carried out with the BOPfox code [18].

\section{Structure map}

In order to illustrate the prediction of TCP phase stability with the structure map, we discuss a set of binary systems $A B$. In particular, we analyse the combinations of average valence-electron concentration $N_{A} / N_{B}$ equal to $5 / 6,5 / 7$, or $5 / 8$ and of relative volume difference $\overline{\Delta V / V}$ equal to $0.1,0.2$, or 0.3 . These choices are close to the corresponding values of, e.g., $\operatorname{Cr}-\operatorname{Re}\left(N_{A} / N_{B}=6 / 7\right.$, $\overline{\Delta V / V} \approx 0.2), \mathrm{Nb}-\operatorname{Re}\left(N_{A} / N_{B}=5 / 7, \overline{\Delta V / V} \approx 0.2\right)$, or Mo-Co $\left(N_{A} / N_{B}=5 / 9, \overline{\Delta V / V} \approx 0.3\right)$ The relation of a binary system to the structure map is given by the parabola

$$
y(\bar{N})=\overline{\Delta V / V}-\left(\frac{N_{A}+N_{B}}{2}-\bar{N}\right)^{2} \frac{\overline{\Delta V / V}}{\left(\frac{N_{A}-N_{B}}{2}\right)^{2}}
$$

that corresponds to the variation of chemical composition. The parabolas obtained for the above combinations of $\bar{N}$ and $\overline{\Delta V / V}$ are indicated in Fig. 2. For each set, the prediction of TCP phases is then given by identifying the intersection of the corresponding parabola with the areas of TCP phase stability given by the polygonal regions in Fig. 2. The TCP phases observed for the above sets are compiled in Tab. 1.

\begin{tabular}{c|c|cccc|cc|cccc}
\multicolumn{5}{c|}{$A_{x} B_{y}$} & \multicolumn{5}{c|}{$x>y$} & \multicolumn{4}{c}{$x<y$} \\
\hline \multicolumn{3}{c|}{} & $\mathrm{A} 15$ & $\sigma$ & $\chi$ & $\mu$ & Laves & $\mu$ & $\chi$ & $\sigma$ & $\mathrm{A} 15$ \\
\hline $6 / 7$ & 0.1 & $\mathrm{X}$ & $\mathrm{X}$ & $\mathrm{X}$ & - & - & - & $\mathrm{X}$ & $\mathrm{X}$ & $\mathrm{X}$ \\
& 0.2 & $\mathrm{X}$ & $\mathrm{X}$ & - & - & $\mathrm{X}$ & $\mathrm{X}$ & $\mathrm{X}$ & $\mathrm{X}$ & $\mathrm{X}$ \\
& 0.3 & $\mathrm{X}$ & $\mathrm{X}$ & - & - & $\mathrm{X}$ & $\mathrm{X}$ & $\mathrm{X}$ & $\mathrm{X}$ & $\mathrm{X}$ \\
\hline $5 / 7$ & 0.1 & $\mathrm{X}$ & $\mathrm{X}$ & - & - & - & - & $\mathrm{X}$ & $\mathrm{X}$ & $\mathrm{X}$ \\
& 0.2 & - & - & - & - & $\mathrm{X}$ & - & $\mathrm{X}$ & $\mathrm{X}$ & $\mathrm{X}$ \\
& 0.3 & - & - & - & - & $\mathrm{X}$ & $\mathrm{X}$ & $\mathrm{X}$ & $\mathrm{X}$ & $\mathrm{X}$ \\
\hline $5 / 9$ & 0.1 & $\mathrm{X}$ & $\mathrm{X}$ & $\mathrm{X}$ & - & - & - & - & $\mathrm{X}$ & - \\
& 0.2 & $\mathrm{X}$ & $\mathrm{X}$ & - & $\mathrm{X}$ & $\mathrm{X}$ & $\mathrm{X}$ & - & - & - \\
& 0.3 & $\mathrm{X}$ & $\mathrm{X}$ & - & - & $\mathrm{X}$ & - & - & - & -
\end{tabular}

Table 1: Possible TCP phase stability for the combinations of average bandfilling and size-difference factor shown in Fig. 2. The symbols $\mathrm{X}$ and - indicate whether a particular TCP phase is expected to be stable or unstable, respectively.

This table gives an indication which TCP phases can be expected for the different binary systems. The TCP phases observed in experiment often exhibit a more narrow range of chemical composition than the intersection of the parabola with the TCP areas in the structure map would suggest. Comparing the TCP stability of different binary systems at constant $N_{A} / N_{B}$ or constant $\overline{\Delta V / V}$ allows us to identify the influence of differences in atomic size or average valence-electron concentration on TCP stability: The Laves phases and the $\mu$ phase are stable only for sufficiently large values of the volume-difference factor $\overline{\Delta V / V}$, regardless of $N_{A} / N_{B}$. Whereas we find Laves phases for both composition ranges of $x>y$ and $x<y$ in $A_{x} B_{y}$ with $V_{A}>V_{B}$, the $\mu$ phase is predominantly observed for $x<y$. The changes in the stability of the phases A15, $\sigma$ and $\chi$ are mostly determined by the valence-electron concentrations of the constituents with an increasing influence of $\overline{\Delta V / V}$ for increasing difference between $N_{A}$ and $N_{B}$. Such an analysis based on the 


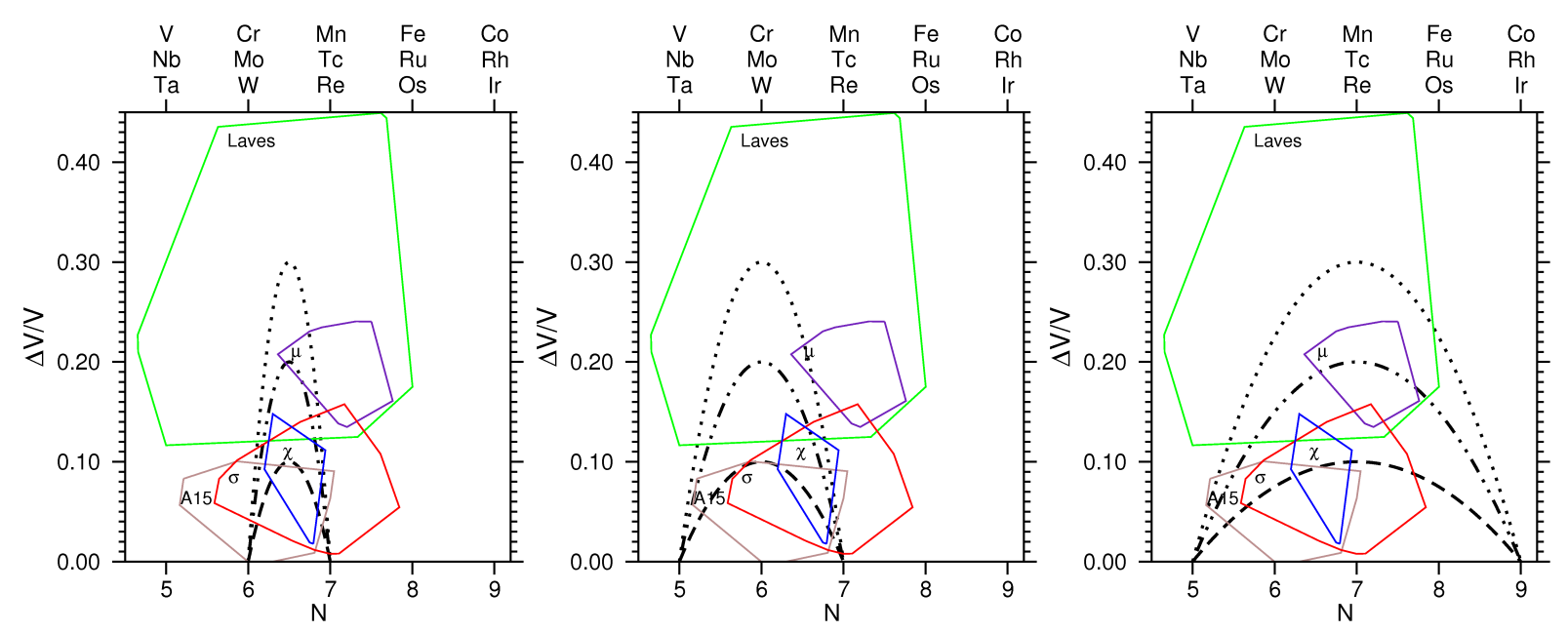

Figure 2: Possible TCP phase stability in binary systems corresponding to alloying elements from group VI-VII (left), V-VII (middle) and V-IX (right). The parabolas indicate varying chemical composition for a given binary alloy. The relative volume differences range from 0.1 (dashed parabolas), over 0.2 (dash-dotted parabolas) to 0.3 (dotted parabolas). The intersection of a particular parabola with the polygonal regions of expected TCP phase stability leads to Tab. 1.

empirical structure-map confirms the previously observed distinct groups of TCP phases [4, 19].

\section{Elemental TCP phases}

In order to isolate the effect of average valence-electron concentration $\bar{N}$, we determined the formation energy of all TCP phases with density-functional theory (DFT), with tight-binding (TB) and with bond-order potential (BOP) calculations. In the DFT calculations the structures are fully relaxed, in the TB and BOP calculations the structures are scaled for a comparison within the structural energy difference theorem as described in more detail in Ref. [3]. The formation energies for the TCP phases of the $5 d$ elements in the middle of the TM series, relative to the formation energy of fcc, are compiled in Fig. 3.

The DFT results for the TM elements (left panel in Fig. 3) show that the TCP phases A15, $\sigma$ and $\chi$ are in close competition with the bcc and the fcc phase at the centre of the band. This regime of stability at half-full band is in line with the structure map of experimentally observed TCP phases (Fig. 1). At the transition from bcc to fcc, the sequence of $\mathrm{A} 15 \rightarrow \sigma \rightarrow \chi$ with increasing $\bar{N}$ observed in DFT is also found experimentally. In the DFT results for the TM elements, we also find the Laves phases to be rather high in energy, which is consistent with the above observation that additional contributions from differences in atomic size are required for their stabilisation. These additional contributions will be addressed in the next section on DFT results for binary TM systems.

Regarding the TB results (middle panel in Fig. 3) we find very good agreement with the DFT results, given that we use a simple canonical TB model and an approximation for the energy differences. Even more clearly than DFT and the structure map, the TB results indicate several distinct groups of TCP phases with phases that behave similarly [4]: C14/C15/C36, $\mu / \mathrm{M}, \mathrm{R} / \mathrm{P} / \delta, \sigma / \mathrm{A} 15$ and $\chi$.

This formation of groups can be further investigated by BOP calculations (right panel in Fig. 3) that allow us to decompose the energy into contributions from different moments. Each additional moment corresponds to an improved description of the electronic density-ofstates through a more far-sighted sampling of the crystallographic environment of each atom. The lowest-order of approximation with only two moments is equivalent to the Finnis-Sinclair potential. By taking into account the sixth moment, the analytic BOP reproduces the structural trend from hcp-bcc-hcp-fcc across the non-magnetic $4 \mathrm{~d}$ and $5 \mathrm{~d}$ transition metal (TM) series [20, 19]. At a level of 8 moments, the analytic BOP quantitatively re- 


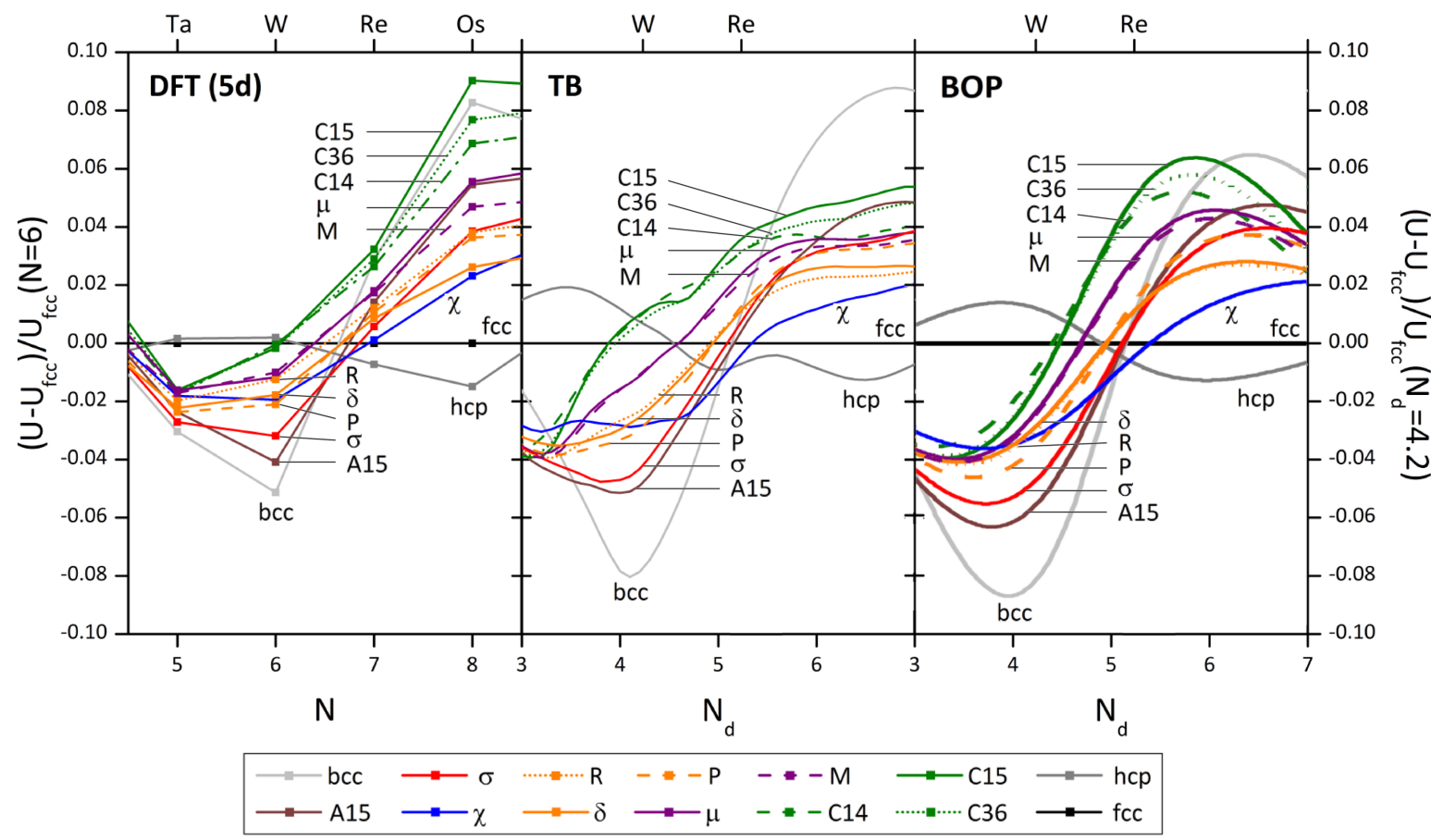

Figure 3: Structural energy difference of TCP phases in the middle of the transition-metal series [4] with respect to fcc-W. The trends of structural stability are conserved during coarse-graining the description of the electronic structure from density-functional theory (left) to a canonical tight-binding model (middle) and further on to analytic bond-order potentials (right).

produces the structural trend of TCP phases observed by TB calculations [4]. Despite the smoother variation of the structural energy differences, the BOP calculations reproduce the groups of TCP phases predicted by the TB calculations. A moments analysis of the BOP calculations shows that the division between $\mathrm{A} 15 / \sigma / \chi$ and the $\mu / \mathrm{C} 14 / \mathrm{C} 15 / \mathrm{C} 36$ phases is related to the bimodality of the density of states caused by distortions compared to ideal Frank-Kasper coordination polyhedra [4].

Currently, we are employing recently developed material-specific bond-order potentials [17] to identify commensurate interfaces between TCP phases and the cubic phases bcc and fcc. Therefore, we optimise the atomistic geometry of the interface with respect to the number of atoms based on the atomically resolved binding energy given by the BOP. An example is given in Fig. 4 for the case of an A15-bcc interface. By optimising the volume of the simulation cell and relaxing the atomic positions, we obtained an interface energy of a few $10 \mathrm{meV} / \AA^{2}$ that needs to be verified by DFT calculations.

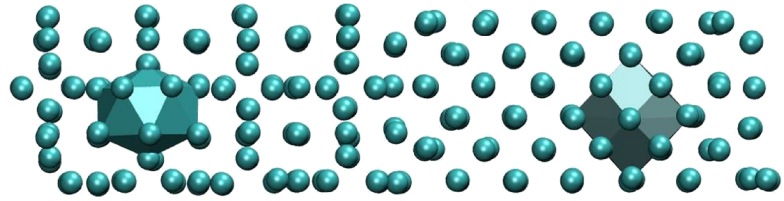

Figure 4: Commensurate interface between A15 and bcc in the crystallographic plane (001) after optimisation of the interface energy with respect to the number of atoms and after atomic relaxation using analytic bond-order potentials.

Ongoing work includes molecular-dynamic simulations for such interfaces with analytic bond-order potentials where we find that this interface is dynamically stable even at elevated temperatures. The analytic BOPs offer sufficient computational efficiency to carry out such simulations of an interface over extended time-scales. They also enable us to routinely study large systems with several 
thousand atoms like, e.g. simulations cells with dislocations. Such systems are currently under investigation using the recently developed material-specific TB models [17] for Mo, Nb, Ta and $\mathrm{W}$. As a preliminary result we show the atom-resolved von-Mises strain invariant in Fig. 5. The detailed analysis of the core structure and its

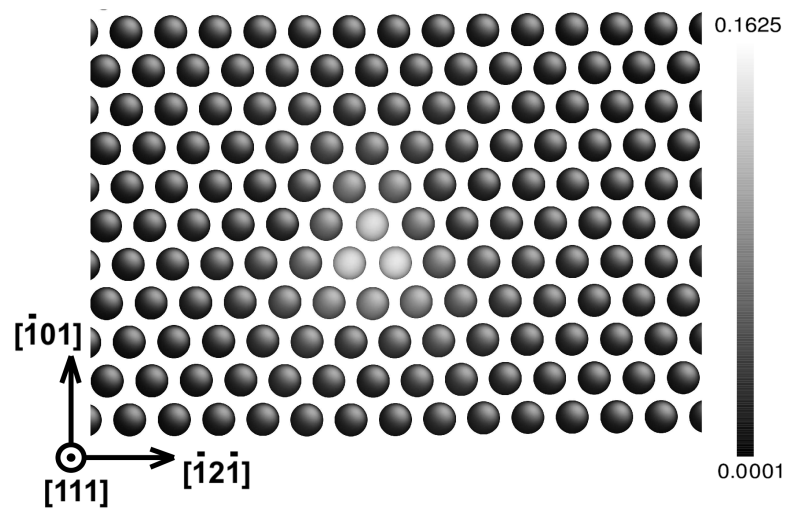

Figure 5: Atom-resolved von-Mises strain invariant for a screw dislocation along the [111] direction in bcc-Ta as obtained with a recently developed analytic bond-order potential [17].

temperature-stability is subject of ongoing investigations.

\section{Binary TCP phases}

In order to assess the influence of differences in atomic size on the structural stability of binary systems, we carried out high-throughput DFT calculations for the TCP phases A15, $\sigma, \chi, \mu, \mathrm{C} 14, \mathrm{C} 15$ and $\mathrm{C} 36$ in the binary systems $\mathrm{V} / \mathrm{Nb}-\mathrm{Ta}, \mathrm{Nb} / \mathrm{Mo}-\mathrm{Ru}, \mathrm{V} / \mathrm{Cr} / \mathrm{Nb} / \mathrm{Mo}-\mathrm{Re}$, $\mathrm{V} / \mathrm{Cr} / \mathrm{Nb} / \mathrm{Mo}-\mathrm{Co}$. In the following we discuss only some of the systems, the full set of results will be given elsewhere $[12,21]$. The relative stability of the different structures and stoichiometries is given by their respective heat of formation $\Delta H_{f}$ per atom given by

$$
\Delta H_{f}=\frac{E_{\mathrm{AB}}-N_{\mathrm{A}} E_{\mathrm{A}}-N_{\mathrm{B}} E_{\mathrm{B}}}{N_{\mathrm{AB}}} .
$$

as the total energy difference of the binary system $E_{\mathrm{AB}}$ to the elemental ground-states $E_{\mathrm{A}}$ and $E_{\mathrm{B}}$. The number of atoms in the binary system and in the elemental groundstates is denoted as $N_{\mathrm{AB}}$ and $N_{\mathrm{A}}, N_{\mathrm{B}}$, respectively. For all phases we considered all possible sub-lattice occupancies of the inequivalent lattice sites. This leads to 4 configurations for $\mathrm{A} 15$ and $\mathrm{C} 15,8$ for $\mathrm{C} 14,16$ for $\chi$, and
32 for $\sigma, \mu$ and C36. In addition to the TCP phases, we also considered ordered fcc, bcc, and hcp structures. For each phase we determined the convex hull individually, as shown in Fig. 6 for Mo/Nb-Re and in Fig. 6 for $\mathrm{Mo} / \mathrm{Nb}-\mathrm{Ru}$.
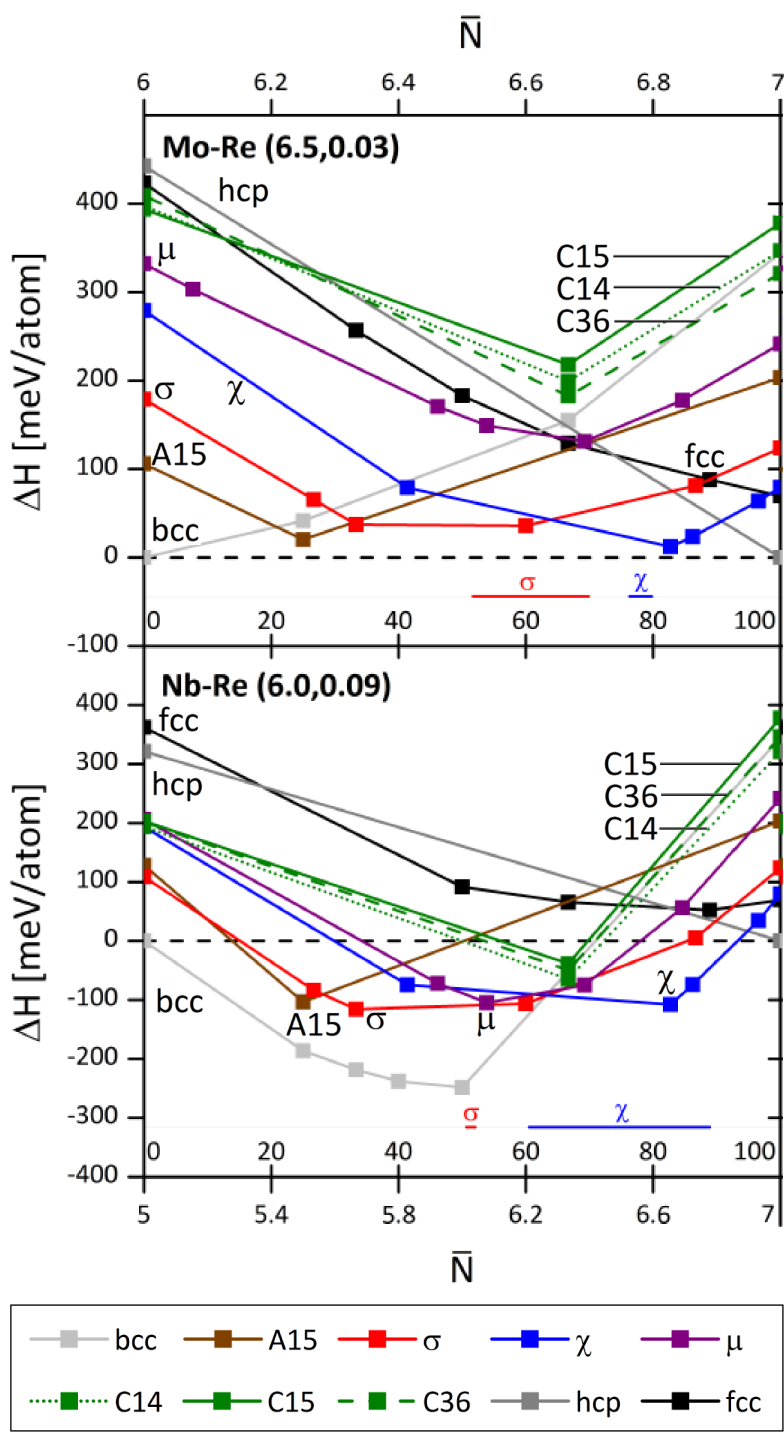

Figure 6: Heat of formation of bcc, fcc, hcp and the TCP phases A15, $\sigma, \chi, \mu, \mathrm{C} 14, \mathrm{C} 15, \mathrm{C} 36$ as obtained by DFT calculations [4] for the systems Mo-Re (top) and Nb-Re (bottom). The experimentally observed phases are indicated as bars, the structure map coordinates $\bar{N}$ and $\overline{\Delta V / V}$ are given in brackets. 
For Mo-Re (top panel of Fig. 6) with $\bar{N}=6.5$ and $\overline{\Delta V / V}=0.03$ we find positive values of the heat of formation for all TCP phases at all compositions. Hence, at a temperature of $0 \mathrm{~K}$, these phases are unstable against phase separation to the elemental ground-states. However, the minima in the convex hull of $\sigma$, and $\chi$ correspond to chemical compositions where these phases are also observed experimentally. The $\mu$ and Laves phases with significantly higher heat-of-formation are unstable as expected from the small size factor difference. For Nb-Re (bottom panel in Fig. 6) the $\mu$ and the Laves phases show negative values of heat-of-formation as expected from the sizeable volume difference of $\overline{\Delta V / V}=0.09$. The structural trend of the TCP phases is unchanged A15 $\rightarrow \sigma \rightarrow \chi$.

The Mo-Ru system (top panel of Fig. 7) with $\overline{\Delta V / V}=$ 0.06 is very similar to Mo-Re: The TCP phases show positive formation energies and the same sequence of most stable TCP phases of $\mathrm{A} 15 \rightarrow \sigma \rightarrow \chi$. A system with a larger volume difference of $\overline{\Delta V / V}=0.13$ is $\mathrm{Nb}-\mathrm{Ru}$ (bottom panel of Fig. 7). The heat-of-formation shows a close competition of the $\mu$ phase with $\mathrm{A} 15, \sigma$, and $\chi$, as well as of the Laves phases with hcp. The experimental phase diagram shows none of these closely competing TCP phases, but only the ordered $\mathrm{L}_{2}$.

The observed discrepancies between the DFT results corresponding to $\mathrm{T}=0 \mathrm{~K}$ and experimentally determined phase diagrams at elevated temperatures indicates the importance of entropic contributions to the relative phase stability that have been observed earlier for ReMo/W [22]. In fact, further studies [12] show that the TCP phases A15, $\sigma$, and $\chi$ can also be stabilised in the systems Re-W/Ta/V despite positive heat-of-formation obtained by DFT. Regarding magnetic contributions, we find only a minor effect on TCP stability for the systems $\mathrm{Co}-\mathrm{Cr} / \mathrm{Mo} / \mathrm{V} / \mathrm{Nb}$ systems that we investigated with similar DFT calculations [21].

\section{Conclusions}

We summarised our recent efforts on understanding the structural stability of topologically close-packed phases in transition-metal compounds. The experimental knowledge on TCP phases was cast in a structure map that allows us to predict the stability of TCP phases even for multi-component systems, based on the average valenceelectron concentration $\bar{N}$ and the composition-dependent relative volume difference $\overline{\Delta V / V}$ that are determined from the constituent elements.

The results are analysed by applying a hierarchy of electronic structure methods. For TCP phases of elemental TMs we find a consistent description of the trends in TCP

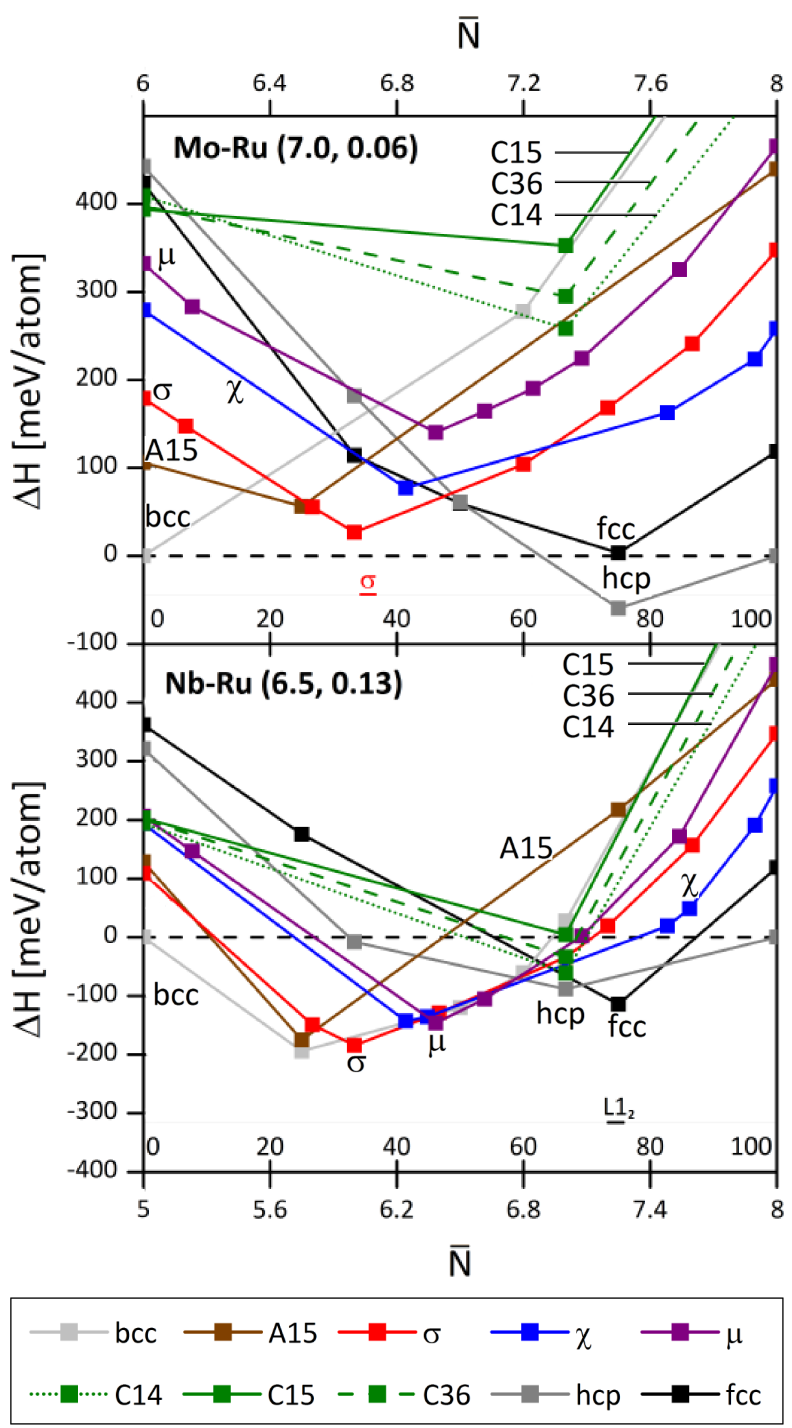

Figure 7: Heat of formation of bcc, fcc, hcp and the TCP phases A15, $\sigma, \chi, \mu, \mathrm{C} 14, \mathrm{C} 15, \mathrm{C} 36$ as obtained by DFT calculations [4] for the systems Mo-Ru (top) and Nb-Ru (bottom). The experimentally observed phases are indicated as bars, the structure map coordinates $\bar{N}$ and $\overline{\Delta V / V}$ are given in brackets.

stability with density-functional theory, tight-binding and bond-order potential calculations. In particular, we show that the average valence-electron concentration stabilises A15, $\sigma$, and $\chi$ but destabilises $\mu$ and the Laves phases 
at the centre of the TM series. The effect of the relative size factor is illustrated by comparing DFT calculations for the binary alloys Mo-Re, Mo-Ru, Nb-Re, and Nb-Ru. The DFT heat-of-formations show that in the system with the largest $\overline{\Delta V / V}, \mathrm{Nb}-\mathrm{Re}$, the $\mu$ and the Laves phases compete closely with the A15, $\sigma$, and $\chi$.

The comparison of the $\mathrm{T}=0 \mathrm{~K}$ DFT calculations to experimental observations highlight the role of entropy for the stability of TCP phases. Molecular-dynamic simulations with analytic BOP open the way to further investigate the stability of interfaces between TCP phases and cubic phases like bcc and fcc at elevated temperatures.

\section{Acknowledgements}

We are grateful to our collaboration partners in the Alloys By Design consortium, in particular Roger Reed, Cathie Rae and Nils Warnken. Part of this work was funded by the Engineering and Physical Sciences Research Council (EPSRC) of the United Kingdom. We acknowledge financial support through ThyssenKrupp AG, Bayer MaterialScience AG, Salzgitter Mannesmann Forschung GmbH, Robert Bosch GmbH, Benteler Stahl/Rohr GmbH, Bayer Technology Services GmbH and the state of North-Rhine Westphalia as well as the EU in the framework of the ERDF.

\section{References}

[1] A. K. Sinha. Prog. Mat. Sci., 15:79, 1973.

[2] C. M. F. Rae and R. C. Reed. Acta. mat., 49:4113 2001.

[3] B. Seiser, R. Drautz, and D. G. Pettifor. Acta mat., 59:749, 2011.

[4] B. Seiser, T. Hammerschmidt, A. N. Kolmogorov, R. Drautz, and D. G. Pettifor. Phys. Rev. B, 83: $224116,2011$.

[5] G. Kresse and J. Hafner. Phys. Rev. B, 48:13115, 1993.

[6] G. Kresse and J. Furthmüller. Comput. Mat. Sci., 6: $15,1996$.

[7] G. Kresse and J. Furthmüller. Phys. Rev. B, 54: 11169, 1996.

[8] P. Blöchl. Phys. Rev. B, 50:17953, 1994.

[9] H. J. Monkhorst and J. D. Pack. Phys. Rev. B, 13: $5188,1976$.
[10] F. D. Murnaghan. Finite Deformation of an Elastic Solid. Dover, New York, 1951.

[11] F. Birch. J. Geophys. Res., 57:227, 1952.

[12] M. Palumbo, S. G. Fries, T. Hammerschmidt, R. Drautz, T. Abe, J.-C. Crivello, and J.-M. Joubert. in preparation: Tolerance of phase stability and site occupancy to numerical accuracy in first principles based phase diagrams of TCP phases in Re-X systems $(X=T a / V / W)$.

[13] T. Hammerschmidt and R. Drautz. In J. Grotendorst, N. Attig, S. Blügel, and D. Marx, editors, NIC Series 42 - Multiscale Simulation Methods in Molecular Science, page 229. Jülich Supercomputing Centre, 2009.

[14] R. Drautz and D. G. Pettifor. Valence-dependent analytic bond-order potential for transition metals. Phys. Rev. B, 74:174117, 2006.

[15] D. G. Pettifor. J. Phys. F: Metal Phys., 7:613, 1977.

[16] O. K. Andersen, W. Klose, and H. Nohl. Phys. Rev. B, 17:1209, 1978 .

[17] M. Cak, T. Hammerschmidt, M. Mrovec, V. Vitek, and R. Drautz. in preparation: Analytic bond-order potentials for bcc transition metals: Parametrisation and comparison to numerical bond-order potentials.

[18] T. Hammerschmidt, B. Seiser, M. E. Ford, D. G. Pettifor, and R. Drautz. to be published: BOPfox program for tight-binding and bond-order potential calculations.

[19] T. Hammerschmidt, B. Seiser, R. Drautz, and D. G. Pettifor. In R. C. Reed, K.A. Green, P. Caron, T.P. Gabb, M.G. Fahrmann, E.S. Huron, and S.R. Woodward, editors, Superalloys 2008, page 847. The Metals, Minerals and Materials Society, 2008.

[20] P. E. A. Turchi. Mat. Res. Soc. Symp. Proc., 206: $265,1991$.

[21] T. Hammerschmidt, A. Bialon, B. Seiser, R. Drautz, and D. G. Pettifor. in preparation: High-throughput ab-initio calculations of topologically close-packed phases in binary transition-metal compounds.

[22] J.-C. Crivello and J.-M. Joubert. J. Phys.: Cond. Mat., 22:035402, 2010. 